\title{
Lung Cancer Prevention and Therapy Using the JinFuKang Herbal Mixture
}

\author{
Lijing Jiao $^{1,2} \cdot$ Yian Wang ${ }^{2} \cdot \operatorname{Ling} \mathrm{Xu}^{1} \cdot \mathrm{Ming} \mathrm{You}^{2}$
}

Published online: 8 May 2015

(C) Springer International Publishing AG 2015

\begin{abstract}
Traditional Chinese Medicine (TCM) plays an indispensable role in China for both cancer prevention and adjuvant treatment in platinum-based chemotherapy. A number of naturally occurring products from Chinese herbal extracts have shown inhibitory effects against carcinogenesis. One example is JinFuKang which is a mixture of 12 Chinese herbs and has been used widely in China for the prevention and treatment of lung cancer. JinFuKang has been shown to inhibit tumor growth in human lung and liver cancer xenograft mouse models. Among all the herbal agents that contain the Astragalus membranaceus (Fisch), JinFuKang was found to be effective in improving the 24-month survival of lung cancer patients in combination with platinum-based chemotherapy. This review article will discuss the application of JinFuKang to cancer prevention and treatment.
\end{abstract}

Keywords Traditional Chinese Medicine $\cdot$ Chinese herbs · JinFuKang · Cancer · Prevention · Therapy

$\begin{array}{ll}\text { Abbreviations } \\ \text { ACF } & \text { Aberrant crypt foci } \\ \beta \text {-Ep } & \beta \text {-Endorphin } \\ \text { BUN } & \text { Blood urea nitrogen }\end{array}$

This work was supported by Longhua Medical Project, No. LYTD-24 (You).

This article is part of the Topical Collection on Cancer Chemoprevention

Ming You

myou@mcw.edu

1 Longhua Hospital, Shanghai University of Traditional Chinese Medicine, 725 South Wanping Rd., Shanghai 200032, China

2 Cancer Center and Department of Pharmacology and Toxicology, Medical College of Wisconsin, 8701 Watertown Plank Road, Milwaukee, WI 53226, USA

\begin{tabular}{|c|c|}
\hline $\mathrm{CR}$ & Complete remission \\
\hline CRE & Serum creatinine \\
\hline CTL & Cytotoxic T lymphocyte \\
\hline CTX & Cytoxan \\
\hline DDP & Di-N-Decyl phthalate \\
\hline $\mathrm{DMH}$ & 1, 2-Dimethyl hydrazine \\
\hline E2 & Estradiol \\
\hline EGFR & Epidermal growth factor receptor \\
\hline EGFR-TKIs & $\begin{array}{l}\text { Epidermal growth factor } \\
\text { receptor-tyrosine kinase inhibitors }\end{array}$ \\
\hline GI & Gastrointestinal \\
\hline GP & Gemcitabine/cisplatin \\
\hline GPT & Glutamate pyruvate transaminase \\
\hline IL-2 & Interleukin-2 \\
\hline JFK & JinFuKang \\
\hline KPS & Karnofsky Performance Status \\
\hline LAK & Lymphokine-activated killer cells \\
\hline LRP & Lung resistance protein \\
\hline MAP & Mitomycin, doxorubicin, and cisplatin \\
\hline MRP & Multidrug resistance associated protein \\
\hline $\mathrm{NC}$ & No change \\
\hline NK & Natural killer \\
\hline NS & Normal saline \\
\hline NSCLC & Non-small cell lung cancer \\
\hline OKT4+ & Helper/inducer T cells \\
\hline $\mathrm{PD}$ & Progression of disease \\
\hline P-EGFR & Phosphorylated-EGFR \\
\hline PLT & Platelet \\
\hline PR & Partial remission \\
\hline $\mathrm{RCT}$ & Randomized controlled trial \\
\hline TCM & Traditional Chinese Medicine \\
\hline Th cells & T helper cells \\
\hline Th1 & $\mathrm{T}$ helper 1 \\
\hline Th2 & T helper 2 \\
\hline VEGF & Vascular endothelial growth factor \\
\hline WBC & White blood cell \\
\hline
\end{tabular}




\section{Introduction}

JinFuKang was originally developed in Shanghai Longhua Hospital, one of the largest traditional Chinese medicine hospitals in China $[1,2,3 \cdot \bullet]$. In addition to the extensive clinical use of JinFuKang as an adjuvant therapy in cancer patients in China $[1,2,3 \cdot \bullet, 4 \bullet]$, JinFuKang has been shown to inhibit tumor growth in animal models by reducing tumor volume in nude mice receiving xenografts of human lung and liver cancer cells $[5,6 \bullet]$. Among all the herbal agents that contain the herb Astragalus membranaceus (Fisch), JinFuKang was found to be one of the most effective agents in improving survival of lung cancer patients as an adjunctive therapy given with platinum-based chemotherapy [4•]. Based on these studies, JinFuKang became one of the major herbal formulations dispensed to lung cancer patients in China. JinFuKang was approved by the Chinese State Food and Drug Administration in 1999 for the treatment of lung cancer (State Approval Number Z19991043). Although the specific constituents of JinFuKang are a trade secret, the individual components and their proportions in the mixture have been previously disclosed (see Table 1 for details) [7••]. JinFuKang is formulated as syrup sealed in glass bottle. Even though the active ingredients in JinFuKang are not known at this moment, their pharmacological, pharmacokinetic, and biopharmaceutical properties have been reported $[6 \bullet, 8,9 \bullet, 10]$. These results also suggest that JinFuKang could be a potent cancer chemopreventive agent, and future preclinical and clinical studies should explore this important area.

\section{Preclinical Studies}

JinFuKang has undergone a series of preclinical studies that revealed inhibition of cancer cell growth as well as enhancing immune function (see Tables 2 and 3). The mechanisms include improvement of the T cell immunity $[12,13]$, reversion of drug resistance [16], inhibition of cancer cell proliferation [15], and induction of apoptosis [19].

JinFuKang prevented colon carcinogenesis in animal models and, thus, could be further developed in colon cancer prevention. In the mouse model of 1,2-dimethylhydrazine (DMH)-induced aberrant crypt foci (ACF), JinFuKang reduced the number of proliferating cells significantly $[1,2]$. In KunMing mice implanted with liver cancer cells, JinFuKang at a dose of $75 \mathrm{~g} / \mathrm{kg} /$ day inhibited liver cancer cell growth by $54.1 \%$ compared with the control group $(P<0.05)$ [5]. In BALB/c nude mice implanted with a human hepatocellular carcinoma cell line, JinFuKang, at $75 \mathrm{~g} / \mathrm{kg} /$ day, inhibited liver cancer cell growth by $32.9 \%$ when compared with the control group $(P<0.05)$ [5]. This study also found that low dose $(10 \mathrm{~g} / \mathrm{kg} /$ day $)$ of JinFuKang is ideal for increasing the production of interleukin-2 (IL-2) [5]. Modulating immune response has been linked to tumorigenesis and cancer progression. The $\mathrm{T}$ helper cells (Th cells) play an important role in the immune system, particularly in the adaptive immune system. JinFuKang significantly increased the Th cells in C57BL/6 male mice implanted with B16 melanoma cells [14]. Another animal study showed that JinFuKang dramatically enhanced the antitumor immune response by increasing the production of T helper 1 (Th1) cytokines while inhibiting production of classical Th2 cytokines in tumor-bearing mice [13]. In C57BL/6 male mice implanted with Lewis lung adenocarcinoma cells, JinFuKang ( $30 \mathrm{~g} / \mathrm{kg} /$ day) enhanced the cytotoxic $\mathrm{T}$ lymphocyte (CTL) in the tumors and tumor growth was significantly inhibited in the JinFuKang group when compared with the control group $(P<0.01)$ [12]. In vivo studies relying on Lewis lung carcinoma grafts also revealed that JinFuKang inhibits tumor growth, increases weight in mice, elevates natural killer (NK) cell number,

Table 1 Composition of JinFuKang (adapted from reference [7••])

\begin{tabular}{|c|c|c|c|}
\hline Latin name & Chinese name & Plant part & Weight $^{\mathrm{a}}(\mathrm{g})$ \\
\hline Astragalus membranaceus & Huang Qi (黄芪) & Root & 146 \\
\hline Glehnia littoralis & Bei Sha Shen (北沙参) & Root & 146 \\
\hline Asparagus cochinchinensis & Tian Men Dong (天门冬) & Root & 49 \\
\hline Ligustrum lucidum & Nv Zhen Zi (女贞子) & Fruit & 49 \\
\hline Selaginella doederleinii & Shi Shang Bai (石上柏) & Leaf & 146 \\
\hline Paris polyphylla & Chong Lou (重楼) & Root & 73 \\
\hline Epimedium sagittatum & Yin Yang Huo (淫羊蕉) & Leaf & 49 \\
\hline Gynostemma pentaphyllum & Jiao Gu Lan (绞股蓝) & Leaf & 49 \\
\hline Cornus officinalis & Shan Zhu Yu (山荣英) & Leaf & 49 \\
\hline Salvia chinensis & Shi Jian Chuan (石见穿) & Fruit & 146 \\
\hline Ophiopogon japonicus & MaiDong (麦冬) & Root & 49 \\
\hline Trigonella foenum graecum & HuLuBa (葫芦巴) & Seed & 49 \\
\hline
\end{tabular}

${ }^{\mathrm{a}}$ Amounts of materials needed to produce $1 \mathrm{~L}$ of finished product, which is syrup. Each dosage contained $10 \mathrm{~mL}$ 


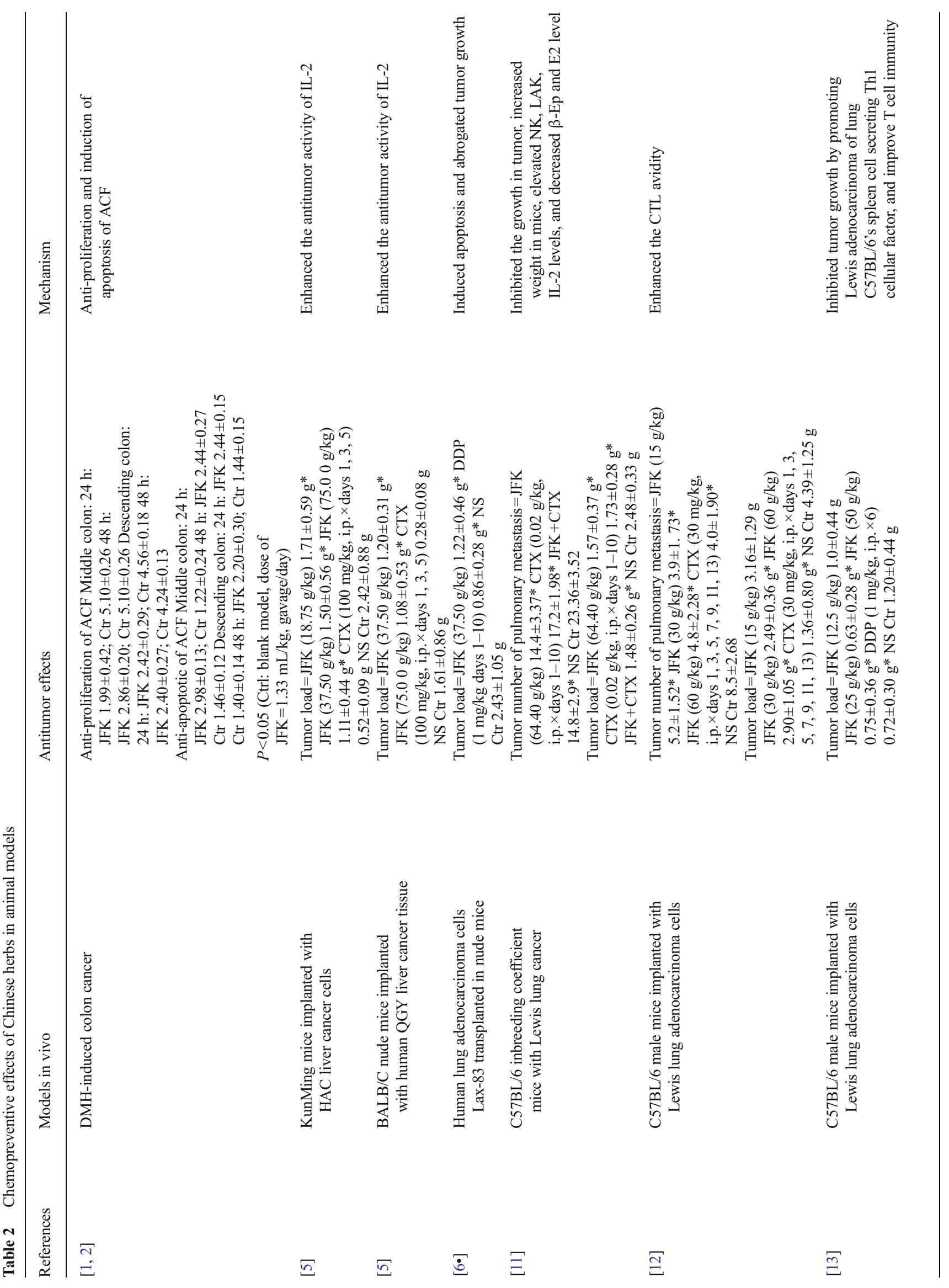


lymphokine-activated killer (LAK) cells, and IL-2 levels, while simultaneously decreasing $\beta$-endorphin ( $\beta$-Ep) and estradiol (E2) levels [11]. JinFuKang also inhibited lung cancer development by $49.2 \%$ versus control in Lax- 83 human lung adenocarcinoma cells transplanted in nude mice [6•]. JinFuKang had inhibitory effect on the metastasis with $33.0 \%$ inhibition in $\mathrm{C} 57 \mathrm{BL} / 6$ male mice implanted with B16 melanoma cells [14].

Many in vitro studies have revealed that JinFuKang may inhibit the proliferation of lung cancer cell lines, induce apoptosis, and alter gene expression. Han et al. [15] found that the inhibitory effects of JinFuKang were due to its inhibition of cell cycle progression in both human lung adenocarcinoma cell line SPC-A-1 and murine Lewis lung cancer cells. JinFuKang significantly reduced the number of S phase cells, increased $\mathrm{G} 2$ and $\mathrm{M}$ phase cells, and inhibited the proliferation of both human lung adenocarcinoma cell line SPC-A-1 and murine Lewis lung cancer cells [12]. JinFuKang's inhibitory effect on cell cycle progression was associated with downregulation of tumor and proliferative markers such as Ki-67 and c-Myc $[8,15]$. JinFuKang also induces apoptosis in lung adenocarcinoma cancer cells LAX-83 by inhibition of $\mathrm{Bcl}-2$ expression and induction of the expression of both Bax and Fas [6॰].

JinFuKang reversed the multidrug resistance of tumor cells. One of the mechanism of the effect on reversing the multidrug resistance is reducing the expression of the drug efflux transporters. A previous study by Sun JL et al. [18] has shown that combination of low-dose JinFuKang and Di$\mathrm{N}$-decyl phthalate (DDP; $320 \mu \mathrm{mol} / \mathrm{L}$ ) can enhance the inhibitory effect of DDP on A549 human lung adenocarcinoma cells from 47.8 to $73.3 \%$. It also reduced the expression of multidrug resistance associated protein (MRP) and lung resistance protein (LRP) at the mRNA level in human lung adenocarcinoma A549 cell line [18]. PC-9 is a cell line derived from a patient with lung adenocarcinoma, harboring an epidermal growth factor receptor (EGFR) exon 19 in-frame deletion [E746-A750] that is highly sensitive to EGFR-TKIs [20]. PC-9/GR is a gefitinib-acquired resistant cell line that was established by chronic exposure of PC-9 cells to the medium with increasing concentrations of gefitinib [21]. JinFuKang also could reverse the resistance to gefitinib in PC-9/GR (resistant cells) cell lines by inducing tumor cell apoptosis, downregulating the expression of phosphorylated-EGFR [16], and enhancing G2/M cell cycle arrest while increasing expression of caspase- 3 and capase-8 [17].

\section{Pharmacokinetics (PK) Studies}

In a reported pharmacokinetic study, changes in docetaxel pharmacokinetics in 21 patients with advanced non-small cell lung carcinoma (NSCLC) were determined. The area under 


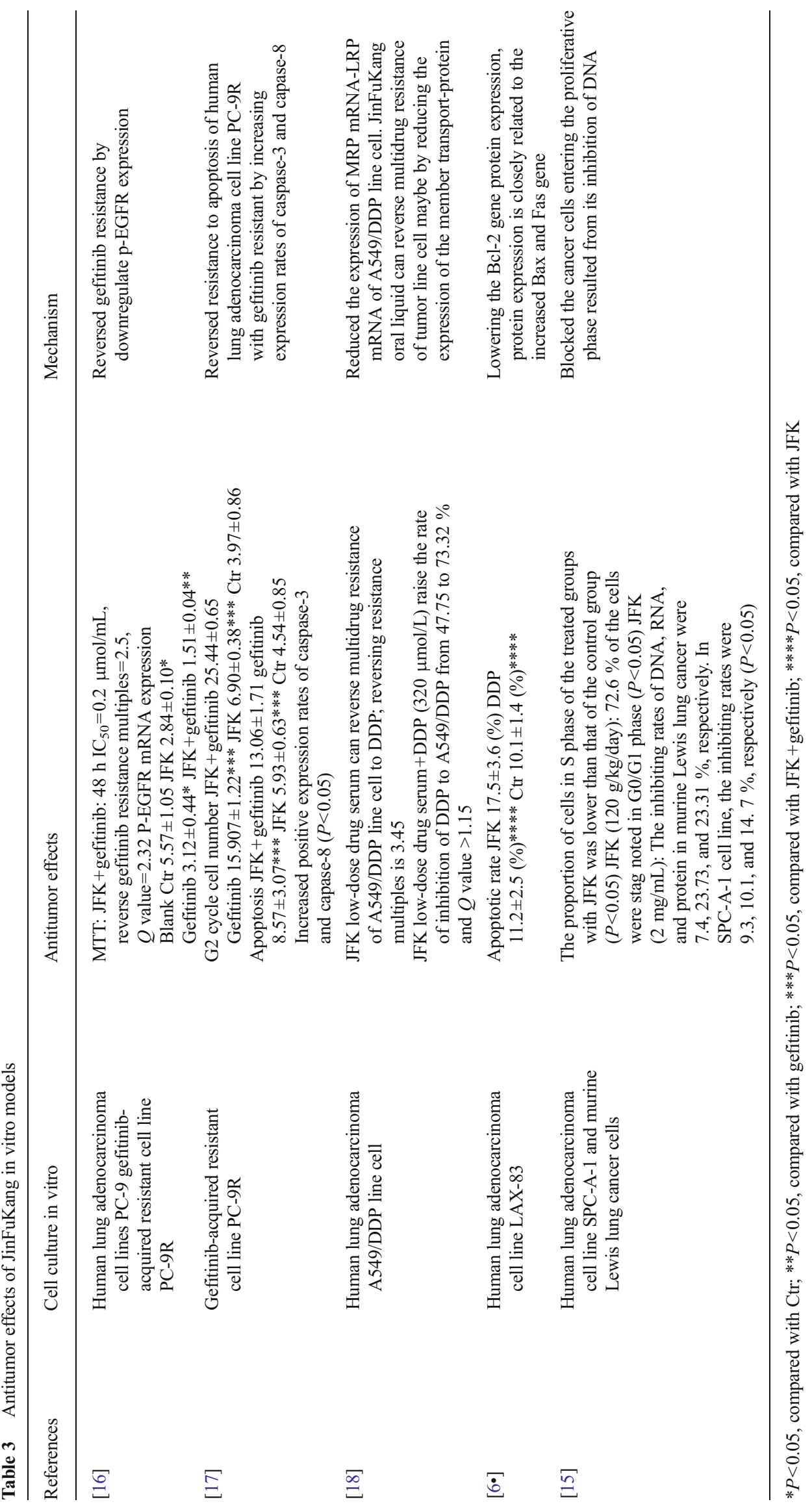


the curve at infinity was estimated to be 104 , whereas clearance rate was estimated $3.8 \mathrm{~L} / \mathrm{h}$. More than $40 \%$ of patients experienced at least a $33 \%$ increased level of docetaxel; a trend is seen although the association is not statistically significant that JinFuKang alters the pharmacokinetics of docetaxel [7••]. In 271 cases of stage II-IV NSCLC patients, there is no change of liver and renal function of 127 cases in the JinFuKang group and 80 cases in the JinFuKang combined with mitomycin, doxorubicin, or cisplatin (MAP) in chemotherapy [9•]. In another larger sample randomized controlled study with 290 cases, this chemotherapy regimen, without the addition of JinFuKang, resulted in a $63.3 \%$ decrease in total white blood cell (WBC) numbers, $26.7 \%$ decrease in platelets (PLT), $14 \%$ increase of glutamate pyruvate transaminase (GPT), and 12.2 and $7.8 \%$ increase of blood urea nitrogen (BUN) and serum creatinine (CRE). Gastrointestinal (GI) reaction and hair loss were 52.2 and $74.4 \%$, respectively. However, when chemotherapy was combined with JinFuKang; many of these adverse events were attenuated. Specifically, the combination of traditional chemotherapy with JinFuKang resulted in a $43 \%$ decrease in WBC, $18 \%$ decrease of PLT, $3 \%$ increase of GPT, and $4 \%$ increase in BUN and CRE levels. GI reaction and hair loss were 17 and $35 \%$, respectively. Not only that it causes no side effects, JinFuKang also reduces toxicity of chemotherapy occurrence rates $[3 \cdot \bullet]$.

\section{Clinical Trials of JinFuKang in Patients With Lung Cancer}

Investigators at Shanghai Longhua Hospital enrolled more than 1000 lung cancer patients in randomized clinical trials (RCT) of JinFuKang, and they have shown that JinFuKang as the first-line therapy could significantly (i) improve the immune function in lung cancer patients $[3 \cdot \bullet, 9 \bullet, 14]$, (ii) improve the quality of life in patients with lung cancer $[3 \cdot \bullet, 9 \bullet$, 14], and also (iii) JinFuKang improves chemotherapeutic effects and decreases toxicities in adjuvant therapy [3••].

As reported by Liu JX et al. [9॰], 271 patients with NSCL Cs were enrolled in a clinical trial from 1991 to 1997 in Shanghai Longhua Hospital. The 271 patients were randomly assigned to three groups. In this study, $88 \%$ of the patients treated with JinFuKang combined with chemotherapy showed a positive response compared with $81 \%$ of the patients treated with JinFuKang alone showed a positive response, and $72 \%$ of the patients treated with chemotherapy alone showed a positive response $(P<0.01)$ [9॰]. A positive response is defined as patients showing complete remission (CR), partial remission (PR), or stable disease (SD) in response to the treatments. Similarly, distal metastasis was observed in $20 \%$ of the patients treated with JinFuKang combined with chemotherapy, in $23 \%$ of patients treated with chemotherapy alone [9•].
A positive response is defined as patients showing CR, $\mathrm{PR}$, or $\mathrm{SD}$ in response to the treatments. The 1-, 2-, 3-, and 4-year survival rates were $73.1,32.0,13.2$, and $13.2 \%$ in the JinFuKang group, respectively; 71.9, 46.4, 29.2, and $23.4 \%$ in JinFuKang combined with the chemotherapy group, respectively; and 37.6, 13.7, 9.7, and $0 \%$ in the chemotherapy group, respectively. In this study, Karnofsky Performance Status (KPS) was elevated in JinFuKang and chemotherapy combined with the JinFuKang treatment groups versus chemotherapy alone. The results showed that JinFuKang could increase KPS scores by increasing survival rate and the quality of life, and enhancing the immune function of NSCLC patients [9॰].

Another study by Liu JX et al. [22] reported that between 1992 and 1994 on 173 lung cancer patients, the response rates were $63.5 \%$ in the JinFuKang group, compared with $60.0 \%$ in the chemotherapy and $71.2 \%$ in the JinFuKang plus chemotherapy group. The 1- and 2-year survival rates were 67.3 and $67.3 \%$ in the JinFuKang group, 40.3 and $0 \%$ in the chemotherapy group, and 66.7 and $66.7 \%$ in the JinFuKang plus chemotherapy, respectively. After treatment, the symptoms, KPS, and immune parameters (NK, LAK, IL-2, $\mathrm{CD}^{+}$, $\left.\mathrm{CD}^{+}\right)$were all improved with the treatment of JinFuKang $(P<0.05)$. The study suggested that JinFuKang could increase survival rate and quality of life and enhance the immune function in NSCLC patients when combined with chemotherapy [22].

In another clinical trial, Wang ZC et al. [23] recruited 115 patients with NSCLCs from 2008 to 2011 . They found that the objective response rate $(\mathrm{CR}+\mathrm{PR}+\mathrm{NC})$ was $68.4 \%$ in the JinFuKang group, compared with $54.3 \%$ in gemcitabine/ cisplatin (GP) group and $78.6 \%$ in JinFuKang combined with gemcitabine/cisplatin $(P<0.05)$. JinFuKang plus gemcitabine/ cisplatin chemotherapy decreased the incidence rates of new metastases, lymph node metastasis, and distant metastasis versus either JinFuKang or gemcitabine/cisplatin treatment alone $(P<0.05)$. In addition, Wang $\mathrm{ZC}$ et al. found that vascular endothelial growth factor (VEGF) blood levels in patients decreased significantly after the treatment of JinFuKang alone or JinFuKang plus gemcitabine/cisplatin $(P<0.05)$ [23]. A clinical study showed that JinFuKang could modulate the ratio of Th1/Th2 cells in patients [24]. The percentage of CTL $\left(\mathrm{CD} 8^{+} \mathrm{CD} 28^{+}\right)$and the ratio of $\mathrm{CTL} / \mathrm{CD} 8^{+} \mathrm{T}$ cells were markedly increased in patients treated with JinFuKang $(P<0.05)$, indicating that JinFuKang could promote activation of CTL and its cytotoxic activity [25].

One of the largest phase II trial was conducted to evaluate the chemotherapeutic efficacy of JinFuKang in 290 mostly $(>72 \%)$ stage II/III NSCLC patients [3••]. Patients that received chemotherapy plus JinFuKang exhibited a $42 \%$ of either complete or partial response, while the patients that received chemotherapy alone showed a $23 \%$ response (either complete or partial response) $(P<0.01)$ indicating that 
JinFuKang has a strong efficacy in treating relatively early stages of lung cancer patients [3••]. In addition, the symptoms, KPS, and immune parameters (NK, IL-2, $\mathrm{CD}^{+}, \mathrm{CD}^{+}, \mathrm{CD}^{+}$/ $\mathrm{CD}^{+}$) were all improved in the JinFuKang group and the JinFuKang plus chemotherapy as compared to the chemotherapy group. The side effects due to toxicity of the chemotherapy were significantly lower in the JinFuKang plus chemotherapy group than the chemotherapy alone group $(P<0.01)$. JinFuKang plus chemotherapy has markedly increased efficacy, and JinFuKang appears to have an ability to decrease the side effects of the chemotherapy [3・•]. JinFuKang appears to have a strong efficacy in treating early stages of lung cancer patients $[3 \cdot \bullet]$.

A more recent clinical trial was conducted to evaluate the chemotherapeutic efficacy of JinFuKang in stage IV NSCLC patients $(>90 \%)[7 \bullet \bullet]$. In this trial, 21 patients received docetaxel (day 1) plus JinFuKang ( $\sim 40 \mathrm{ml}$ t.i.d., or $75 \mathrm{ml} / \mathrm{m}^{2} /$ day, days 4-28). Only 16 patients completed 1 cycle. After 1 cycle, 9 withdrew because of disease progression (7) or toxicity (2) and only 7 completed 2 or more cycles, and none showed improvement suggesting that JinFuKang has limited effects on very late stage (stage IV) NSCLC patients. The trial was considered to be unsuccessful because only 7 patients completed treatments. Since it only enrolled patients with very advanced stages of lung cancer ( $>90$ stage IV patients), the outcome is quite different from the previous large phase II trial $[3 \bullet \cdot$. These studies suggest that JinFuKang is more effective in patients with early stages of lung cancer.

\section{Future Perspectives and Conclusions}

To certain degrees, the cancer-preventive effects of Chinese herbs have been supported by results from laboratory animals, cell cultures, and epidemiological and clinical studies. A typical chemopreventive agent should have an outstanding safety profile, efficacious, and be amenable to oral administration, which makes JinFuKang an ideal chemopreventive agent. In additional to the long-term and safe clinical experiences in humans, JinFuKang is orally active. JinFuKang is one of the most promising traditional Chinese herbs for lung cancer chemoprevention because it has been used for a long period of time in China by a large population with a good safety profile. Several clinical studies have been conducted in China with the largest phase II trial to evaluate the chemotherapeutic efficacy of JinFuKang in 290 mostly (>72\%) stage II/III NSCLC patients [3••]. Patients receiving chemotherapy plus JinFuKang exhibited a response rate of $42 \%$ (either complete or partial response), while the patients receiving chemotherapy alone showed a $23 \%$ response (either complete or partial response) $(P<0.01)$, which indicates that JinFuKang is efficacious addition to therapy in earlier stage disease in lung cancer patients $[3 \bullet \bullet]$.
Similarly, JinFuKang is expected to be effective in a chemoprevention setting. Secondly, it is prepared in a GMP facility (GMP refers to the Good Manufacturing Practice Regulations by the US Food and Drug Administration), allowing the standardization for the preparation of the herbal materials. Although JinFuKang has shown profound effects on lung cancer, further mechanistic studies are urgently needed to understand the basis of its efficacy against some types of cancer. In general, future research efforts to develop herbal mixtures should focus on those with lower toxicity and higher efficacy for specific types of cancer through the inhibition of specific molecular targets or pathways.

\section{Compliance with Ethics Guidelines}

Conflict of Interest Lijing Jiao, Yian Wang, Ling Xu, and Ming You declare that they have no conflict of interest.

Human and Animal Rights and Informed Consent This article does not contain any studies with human or animal subjects performed by any of the authors.

\section{References}

Papers of particular interest, published recently, have been highlighted as:

- Of importance

•- Of major importance

1. Dong L, Zhan GX, WU DZ. Inhibition of dimethyl hydrazine $(\mathrm{DMH})$ induced colorectal cancer in rats by JinFuKang. Chin Tradit Patent Med. 2007;29(7):1061-3.

2. Qiu YP, Su MM, WU DZ, Zhao AH, Liu YM, Jia W. Effect of JinFuKang to experimental precancerous colon lesions and urinary metabolites in rat. China J Chin Mater Med. 2008;33(22):2653-7.

3.• Liu JX, Pan MQ, Li YH, Ye DQ, Guo YH, Li YJ. Clinical study of oral liquid Jin Fu Kang for the treatment of primary non-small cell lung cancer. Tumor (Shanghai). 2001;21(6):463-5. This is one of the largest clinical trials on JinFuKang designed to evaluate the chemotherapeutic efficacy of JinFuKang in stage II/III NSCLC patients. This study showed that patients that received chemotherapy plus JinFuKang exhibited a higher response than chemotherapy alone. The symptoms, Karnofsky Performance Status (KPS), and immune parameters were improved, and the side effects of the chemotherapy were decreased.

4. McCulloch M, See C, Shu XJ, Broffman M, Kramer A, Fan WY, et al. Astragalus-based Chinese herbs and platinum-based chemotherapy for advanced non-small-cell lung cancer: meta-analysis of randomized trials. J Clin Oncol. 2006;24(3):419-30. This study using meta-analysis on Astragalus-based Chinese herbs indicates that Astragalus may increase effectiveness of platinumbased chemotherapy in advanced NSCLC.

5. Li HG, Chen XH, Yao YL, Ren WL. A study of JinFuKang in the treatment of liver cancer. Chin Tradit Herb Drugs. 2000;31(7): 533-5.

6. Sun JL, Liu JX. Effect of “JinFuKang oral solution" on expression of apoptosis related genes of human lung adenocarcinoma cells 
transplanted in nude mice. Shanghai J Tradit Chin Med. 2007;41(10):69-71. This work showed that JinFuKang inhibited lung cancer development by $49.2 \%$ versus control in Lax-83 human lung adenocarcinoma cells transplanted in nude mice. Its molecular mechanism may be related to lower Bcl-2 protein expression, which is closely related to the increased expression of both Bax and Fas.

7.• Cassileth BR, Rizvi N, Deng G, Yeung KS, Vickers A, Guillen S, et al. Safety and pharmacokinetic trial of docetaxel plus an Astragalus-based herbal formula for non-small cell lung cancer patients. Cancer Chemother Pharmacol. 2009;65(1):67-71. Based on a 21-patient clinical trial conducted on stage IV NSCLC patients with 7 patients completed trial-treatment, this paper reported that JinFuKang has limited effects on late-stage (stage IV) NSCLC patients and it did not alter the pharmacokinetics of docetaxel. The individual components and their proportions in the mixture have been disclosed in this article for the first time.

8. Xu L, Liu JX. Effects of Yifei Kangliu oral liquid on cell cycle and protein-nucleic acid synthesis of experimental lung cancer. Chin $\mathrm{J}$ Integr Tradit West Med. 1996;16(8):486-8.

9. Liu JX, Shi ZM, Li HG, Xu ZY, Zhu YW, Zhao LH, et al. Clinical observation on 271 cases of non-small cell lung cancer treated with Yifei Kangliu Yin. Chin J Integr Med. 2001;7(4):247-50. In this study, Karnofsky Performance Status (KPS) was elevated on JinFuKang and chemotherapy combined with JinFuKang treatment groups versus chemotherapy alone. This work showed that JinFuKang increases KPS scores by increasing survival rate and the quality of life, and enhancing the immune function of NSCLC patients.

10. Piao B-K, Li P-W, Liu JX. Experiences and prospects on tumor therapy by integrative Chinese and Western medicine. Zhongguo Zhong Xi Yi Jie He Za Zhi. 2003;23(8):612-5.

11. Zhu HR, Liu JX. Experimental study of "Lung-Benefiting and Tumor-Resisting Drink" on neuroendocrine immunity in mice with lewis pulmonary cancer. Acta Univ Tradit Med Sinen Pharmacol Shanghai. 2000;14(2):44-6.

12. Sun G, Liu JX. The effect of JinFuKang destruction T-lymphocyte function in Lewis lung mice. Hunan J Tradit Chin Med. 2007;02: $92-3$.

13. Sun XY, Sun JL, Liu JX. Effect of Jin Fu Kang oral liquid for Lewis adenocarcinoma of lung C57BL/6's spleen secreting THI/TH2 cellular factor: experimental study. J Liaoning Univ Tradit Chin Med. 2008;10(9):160-2.

14. Xu L, Liu JX. Effect of Yifei Kangliu Yin on lung cancer metastasis and immune function. Chin J Integr Tradit West Med. 1997;17(7): 401-3.

15. Han MQ, Liu JX, Gao H, Chen SX, Zhu YW, Xu L. Effects of Yifei Kangliu oral liquid on cell cycle and protein-nucleic acid synthesis of experimental lung cancer. J Integr Med. 2003;1(3):205-8.

16. Sun XY, Jiang M, Zhang M, Liang YT, Sun JL. The effect of JinFuKang on acquired drug resistance of non-small cell lung adenocarcinoma. J Chin Med Mater. 2014;07:1254-8.

17. Sun XY, Jiang M, Zhang ZT, Zhang W, Xu HY, Liang YT, et al. Effect of JinFuKang oral liquid on apoptosis of human lung adenocarcinoma cell line PC-9R with gefitinib resistant. Liaoning J Tradit Chin Med. 2014;10:2229-32.

18. Sun JL, Sun XY, Liu JX. Effect of Jin Fu Kang oral liquid on the expression of MRP mRNA-LRP mRNA of the human lung adenocarcinoma A549/DDP line cell. J Liaoning Univ Tradit Chin Med. 2009;11(7):210-3.

19. Dong L, Shu D, Wang JH, Lv JP. Selective apoptosis enhancement of JinFuKang on rat colonal crypt. J Taishan Med Coll. 2006;8:712-4.

20. Koizumi F, Shimoyama T, Taguchi F, Saijo N, Nishio K. Establishment of a human non-small cell lung cancer cell line resistant to gefitinib. Int J Cancer. 2005;116(1):36-44.

21. Cheng H, An SJ, Dong S, Zhang YF, Zhang XC, Chen ZH, et al. Molecular mechanism of the schedule-dependent synergistic interaction in EGFR-mutant non-small cell lung cancer cell lines treated with paclitaxel and gefitinib. J Hematol Oncol. 2011;4:5.

22. Liu JX, Shi ZM. Clinical study on treating non-small cell lung carcinoma with JinFuKang oral liquid. J Tradit Chin Med. 1997;38(12):727-9.

23. Wang ZC, Zeng JL, Zhang HL, Li JW. Jin Fu Kang on tumor growth and metastasis and vascular endothelial growth factor of patients with advanced non-small cell lung cancer. Pract J Clin Med. 2011;8(4):148-9.

24. Sun G, Liu JX. The effect of JinFuKang on IL-10 and IFN in patients with lung cancer. Shandong J Tradit Chin Med. 2001;20(12):721-02.

25. Sun G, Liu JX. Influence of JinFuKang on express of T lymphocyte antigen in lung cancer patients' peripheral blood. Liaoning J Tradit Chin Med. 2001;28(5):279-80. 